\title{
The Value of Ultrasound in Characterizing and Determining the Etiology of Ascites
}

\section{Wondim Getnet $^{1 *}$, Tesfaye Kebede ${ }^{1}$, Asfaw Atinafu' ${ }^{1}$, Amir Sultan ${ }^{2}$}

\footnotetext{
OPEN ACCESS

Citation: Natnaael Kidanu Yibalih, Dawit Wolday, Samuel Kinde, Gebru Mulugeta Weldearegay. External Quality Assessment on CD4+ T-Cell Count Using in-House Proficiency Testing Panels for CD4 Count Laboratories in Addis Ababa, Ethiopia. Ethiop J Health Sci. 2019;29(3):383.

doi:http://dx.doi.org/10.4314/ ejhs.v29i3.11

Received: March 18, 2019

Accepted: March 4, 2019

Published: May 1, 2019

Copyright: (C) 2019 Wondim Getnet, et al. This is an open access article distributed under the terms of the Creative Commons Attribution License, which permits unrestricted use, distribution, and reproduction in any medium, provided the original author and source are credited. Funding: Addis Ababa University Competing Interests: The authors declare that this manuscript was approved by all authors in its form and that no competing interest exists.

Affiliation and Correspondence:

${ }^{1}$ Radiology Department, Tikur Anbessa Specialized Hospital, College of Health Science, Addis Ababa University, Addis Ababa, Ethiopia

${ }^{2}$ Internal Medicine Department, Tikur Anbessa Specialized Hospital, College of Health Science, Addis Ababa University, Ethiopia *Email: yisak_wodim@yahoo.com
}

\author{
ABSTRACT
}

BACKGROUND: The aim of this study was to assess the role of transabdominal ultrasonography in characterizing and determining the etiology of ascites in comparison with laboratory ascitic fluid analysis and other methods used to establish the final diagnosis.

METHODS: A prospective descriptive study was conducted on 61 patients with ascites attending outpatient department (OPD) or admitted to wards of Tikur Anbesa Specialized Hospital (TASH) and referred to radiology department for imaging from June 2017 to November 2017. Data were collected following the internationally recommended scanning technique in consecutive bases. The data were analyzed using SPSS version 20. The comparison of ultrasound and laboratory findings with final clinical diagnosis was analyzed using Chi-square test $\left(X^{2}\right)$.

RESULTS: Of 61 patients with ascites enrolled in this study, females were 35(57.4\%) with age range of 16 to 75 and mean age of 43.2 \pm 14.11 . The cause of ascites was established in 59 cases using a combination of clinical, pathological, imaging evidences and tumor markers. However there were two cases who had ascites with indeterminate cause. US suggested the diagnosis in 54(91.5\%) patients. Excluding mixed and indeterminate cases, ultrasound characterized ascites correctly as exudate and transudate in $95 \%$ cases.

CONCLUSION: Ultrasound has significant accuracy to distinguish transudate and exudate ascites and in suggesting the underlying cause. It can be a valuable method of investigation of ascites in places where CT and MRI are not available, and it is the best complement for laboratory investigations on ascites in suggesting the etiology based on ascitic fluid texture and ancillary findings.

KEYWORDS: ultrasound, ascites etiology, ascites characterization, $S A A G$, disease categories

\section{INTRODUCTION}

Ascites is a common clinical problem. Many disease processes may lead to the development of ascites. It may be the first manifestation of a systemic disease or can develop due to local intra-abdominal pathology. Local diseases processes which directly involve the peritoneum and causing ascites include 
infection and malignancy. Systemic diseases that indirectly affecting peritoneum comprises such as liver and renal diseases, heart failure, and hypoproteinaemia $(1,2,3)$.

Cirrhosis is the commonest cause both in developed and developing countries contributing to three-quarter of cases $(3,4)$. Accumulations of blood, urine, chyle, pancreatic secretions, or bile within the peritoneal cavity is the more unusual cause (1).

Ascites has to be detected before characterization, and there must be sensitive means to do so. Physical examination is not very accurate in the diagnosis of ascites. Cattau et al. showed that the overall accuracy of the physical maneuvers was only 58\% (5). Currently, cross sectional imaging is playing a vital role and almost replaced other methods in the detection and evaluation of intraperitoneal fluid collections. They can detect ascites which was not even suspected clinically. As established experimentally, the sensitivity of ultrasound in the detection of fluid has been as little as $100 \mathrm{ml}$ in cadavers and $300 \mathrm{ml}$ in vivo (6).

Traditionally, the ascitic fluid was broadly categorized into transudative and exudative fluids based on the ascites fluid total protein (AFTP). Ascitic fluid was classified as exudates if the AFTP was $25 \mathrm{~g} / \mathrm{L}$ or more and as transudates if the AFTP was less than $25 \mathrm{~g} / \mathrm{L}$; occasionally, $30 \mathrm{~g} / \mathrm{L}$ was used as the level of discrimination. However, many problems and exceptions have been observed in this method of classification. Because of that, a more meaningful system, called the Serum Ascites Albumin Gradient (SAAG), has been developed. This is the difference between the amounts of albumin in the serum and ascitic fluid albumin $(7,8)$.

Several studies showed that SAAG is a test with significant diagnostic accuracy in separating ascites related to portal hypertension with SAAG value of generally $\geq 1.1 \mathrm{~g} / \mathrm{dL}$ from the forms of ascitic fluid caused by non-portal hypertension (SAAG value $=<1.1 \mathrm{~g} / \mathrm{dL})(7-10)$. However, it does not provide specific cause for ascites in either portal hypertension or non-portal hypertension (9). In contrast, imaging plays a significant role in suggesting the definite etiology because of its utility in studying all intra-abdominal and pelvic structures.

Once ascites is detected, the next crucial step is determining the etiology of the ascites which is important for instituting an appropriate treatment plan (2). US and CT have major contributions in identifying ancillary findings that help in suggesting specific diagnosis or narrowing the differential diagnosis.

Certain signs on US and CT are helpful in predicating etiology of ascites. Distribution of ascites (greater sac, lesser sac or both), presence of peritoneal implants, omental cake, and tethered bowel signs are extremely useful in differentiating benign from malignant ascites on imaging $(2,3,11)$. Previous studies showed the important role of ultrasound in characterizing the ascitic fluid as transudate and exudate. It has also a role in defining its etiology in significant number of cases $(2,3,11)$.

It is well-known that small amount of ascitic fluid can be detected with $\mathrm{CT}$ and MRI, but US has several advantages and continues to be the primary screening modality for patients with ascites. Among its advantages are speed and mobility, absence of ionizing radiation, and invulnerability to breathing artifact in dyspneic or minimally cooperative patients. Compared with $\mathrm{CT}$ and MRI, ultrasound is a more valuable method of investigation in low-resource countries, like Ethiopia, because of its wide availability, accessibility, mobility, cheap cost and safety.

Therefore, this prospective descriptive study aimed to determine the diagnostic role of US in the characterization of ascites as exudate and transudate, and determination of its etiology with reference to final diagnosis established with, such as, laboratory diagnostic test, pathology, other cross- 
sectional imaging, laparotomy and clinical course.

If the study demonstrates the presumed diagnostic capacity of ultrasound, it can be used in places where CT and MRI are inaccessible or unavailable. As the result of this, the cost of care born by patients will significantly be reduced

\section{MATERIALS AND METHODS}

This study was conducted from June 25, 2017 to November 30, 2017 at Tikur Anbessa Specialized Hospital (TASH). We have not determined sample size as we initially aimed to include as many patients as possible with the allocated resources and time. Given the nature of the condition we were considering, we would have sufficient cases to answer our research questions. Sixty-one consecutive patients were prospectively enrolled in the study.

Following the internationally recommended scanning technique (12), sonographic examination was done using Sonoscape machine (SSI-800, Nanshan, China) equipped with linear and curvilinear transducers. The sonographic examination was used to study the presence, echotexture, distribution and loculation of ascites. It was also used to detect ancillary findings such as GB wall thickening, omental and peritoneal thickening and/or deposits, tethering and matting of bowel loops as well as the presence of lymphadenopathy.

Immediately after the ultrasound examination within an average of 14 minutes interval), US guided paracentesis was done under sterile conditions. Blood sample was also drawn immediately after the paracentesis. The ascitic fluid and blood samples were sent to the laboratory to determine ascitic albumin, cell count and differential as well as serum albumin and total protein. A portion of ascitic fluid sample was also sent to the pathology unit for cytology. The serum and ascitic albumin values were used to determine the serumascites albumin gradient (SAAG).
The demographic data, ultrasound, laboratory and cytology findings as well as other imaging findings and pertinent clinical data were recorded in a format prepared in advance. The completeness and consistency of the questionnaire for each patient were checked at the time of data collection.

The Ethical clearance for the proposed study was obtained from the Research Ethics Committee of the Department of Radiology, College of Health Sciences, Addis Ababa University. Informed consent was obtained from patients before starting the paracentesis and drawing blood sample.

The colledcted Data were checked for completeness, coded, entered into SPSS version 20 statistical software packages, cleaned and analyzed by the principal investigator. Fisher's exact/chi-square test was used to evaluate the efficacy of US scan in determining the pathologies, and the pathological difference between malignant and benign cases. A p-value of $<0.05$ indicated a statistically significant difference.

\section{RESULTS}

In this study, a total of 61 patients with ascites were included. The age ranges from 16 to 75 with mean age of $43.2 \pm 14.11$. Females constituted 35 (57.4\%). The majority of patients were referred from medical and emergency OPD. Most of the patients, 35 $(57.4 \%)$, referred with clinical presentation of abdominal swelling.

The cause of ascites was established in $59(96.7 \%)$ of the cases using clinical, pathological and imaging evidences. This was not possible for two cases. One was a mother who had caesarean section for indication of worsening preeclampsia one month prior to the US examination. There was no obvious cause for ascites identified on ultrasound scanning, and it was not possible to retrieve her hospital record to find out if there was any clinically attributable cause. The second patient was on workup for suspected breast cancer but no evidence was found on abdominal sonographic study to suggest etiology for ascites. Her hospital record was

DOI: http://dx.doi.org/10.4314/ejhs.v29i3.11 
not also retrieved. No other imaging was done in both cases.

Broadly, etiologies of ascites were classified as benign, malignant, inflammatory and mixed causes. Malignant causes were found in $20(32.9 \%)$ cases, and it was the commonest cause of ascites, followed by chronic liver disease 9(14.8\%) (Table 1). Among malignant causes, ovarian cancer constituted the major part, 11(47.8\%) (Table1). Hepatocellular carcinoma (HCC) was the second commonest cancer cause but included under mixed causes because all cases of HCC occurred on the background of cirrhotic liver. Among 16 mixed causes, $10(62.5 \%)$ were due to HCC which occurred on the background of cirrhotic liver. In such cases, the ascites can be caused either by portal hypertension as a result of cirrhotic liver disease or bland/tumor thrombosis in the presence of primary tumor in the liver or can occur secondary to peritoneal deposits or extra-hepatic intra-abdominal spread of HCC. Table 1 summarizes the specific pathologies included under each etiological category.

Table 1: Specific pathologies under each category with their frequency in 61 patients at TASH, 2017

\begin{tabular}{lll}
\hline Benign cause $=16(26.2 \%)$ & Frequency & Percent \\
CLD \pm PVT & 9 & 14.8 \\
PVT+BCS & 3 & 4.9 \\
Cardiac & 3 & 4.9 \\
Renal & 1 & 1.6 \\
Inflammatory=7 (9.8\%) & 5 & 8.2 \\
Tuberculosis peritonitis & 1 & 1.6 \\
Pyogenic peritonitis & & \\
Malignant cause=20 (32.8\%) & 10 & 16.4 \\
Ovarian cancer & 2 & 3.3 \\
Gastric cancer & 2 & 3.3 \\
GB cancer & 2 & 3.3 \\
Lymphoma & 4 & 6.6 \\
Others & & 16.4 \\
Mixed causes $=17$ (27.9) & 10 & 3.3 \\
CLD+HCC \pm PVT or BCS & 2 & 3.3 \\
Crohn's disease + hypoproteinemia & 2 & 1.6 \\
Tuberculous peritonitis + hypoproteinemia & 1 & 1.6 \\
Hematological malignancy + adult malnutrition & 1 & 1.6 \\
Pancreatic cancer +cardiac & 1 & 3.3 \\
Colonic cancer + hypoproteinemia & 2 & \\
Indeterminate causes (3.3\%) & 61 & \\
Grand total & &
\end{tabular}

$\mathrm{CLD}=$ chronic liver disease; PVT-portal vein thrombosis; BCS-Budd Chiari syndrome; HCC-hepatocellular carcinoma

Ultrasound was able to classify $100 \%$ of benign causes as transudate and $95 \%$ of malignant and inflammatory causes as exudates (Table 2). SAAG was also able to determine $15(94 \%)$ of 16 benign cause and $14(70 \%)$ malignant and inflammatory causes
(Table 3). The overall agreement between the US and SAAG methods of evaluation of ascites was in $49(83.1 \%)$ cases. The agreement is more noticeable in benign causes.

DOI: http://dx.doi.org/10.4314/ejhs.v29i3.11 
Table 2: Crosstabulation of sonographically determined ascitic pattern with disease categories in 61 patients at TASH, 2017.

\begin{tabular}{|c|c|c|c|c|c|}
\hline & & \multicolumn{3}{|c|}{ US determined ascites pattern } & \multirow[t]{2}{*}{ P.value } \\
\hline & & Transudate & Exudate & & \\
\hline Disease & Benign & 16 & 0 & 16 & \multirow{6}{*}{0.000} \\
\hline \multirow{5}{*}{ categories } & Malignant & 1 & 19 & 20 & \\
\hline & Inflammatory & 1 & 5 & 6 & \\
\hline & Mixed & 11 & 6 & 17 & \\
\hline & Indeterminate & 2 & 0 & 2 & \\
\hline & Total & 31 & 30 & 61 & \\
\hline
\end{tabular}

Table 3: Crosstabulation of SAAG determined ascitic pattern with disease categories in 61 patients, at TASH, 2017.

\begin{tabular}{clllll}
\hline & & \multicolumn{2}{l}{$\begin{array}{l}\text { SAAG determined ascites } \\
\text { pattern }\end{array}$} & P. value \\
\cline { 3 - 4 } & & $\begin{array}{l}\text { Transudate } \\
(\geq 1.1 \mathrm{~g} / \mathrm{dl})\end{array}$ & $\begin{array}{l}\text { Exudate } \\
(<1.1 \mathrm{~g} / \mathrm{dl})\end{array}$ & Total & \\
\hline Disease & Benign & 15 & 1 & 16 & \\
categories & Malignant & 6 & 14 & 20 & \multirow{2}{*}{0.000} \\
& Inflammatory & 2 & 4 & 6 & \\
& Mixed & 16 & 1 & 17 & \\
\cline { 3 - 4 } Total & Indeterminate & 2 & 0 & 2 & 61 \\
\hline
\end{tabular}

In addition to characterizing the ascitic fluid, ultrasound was used to investigate visceral and hollow organs as well as lymph nodes of the abdomen and pelvis. The pathological changes in those organs and peritoneal layers can suggest the etiology of ascites. Furthermore, the patency of abdominal vessels including aorta, IVC, portal and hepatic veins and their caliber, and the presence of collaterals and cavernous transformation was checked. Based on composite of findings, ultrasound suggested diagnosis. Taking all evidences US was able to suggest nearly accurate diagnosis in $54(91.5 \%)$ out of 59 cases.

The volume of ascites determined in this study ranged from $14 \mathrm{ml}$ to $9976 \mathrm{ml}$. The volume of ascites was classified into five grades. However, for the purpose of comparison, the frequency of grades $0-2$ and 3-4 were combined and cross tabulated with disease categories (Table 4) and SAAG.

Table 4. Cross tabulation between disease categories and ascitic volume grading in 61 patients at TASH, 2017.

\begin{tabular}{lllll}
\hline \multicolumn{5}{c}{ Graded ascitic volume } \\
\hline $\begin{array}{llll}\text { Disease } \\
\text { categories }\end{array}$ & Grade $0-2$ & Grade 3-4 & Total & P value \\
\hline Benign & $8(50 \%)$ & $8(50 \%)$ & $16(100 \%)$ & \\
Malignant & $9(45 \%)$ & $11(55 \%)$ & $20(100 \%)$ & 0.573 \\
Inflammatory & $5(83.3 \%)$ & $1(16.7 \%)$ & $6(100 \%)$ & \\
Mixed & $8(47 \%)$ & $9(53 \%)$ & $17(100 \%)$ & \\
Indeterminate & $1(50 \%)$ & $1(50 \%)$ & $2(100 \%)$ & \\
\hline
\end{tabular}

DOI: http://dx.doi.org/10.4314/ejhs.v29i3.11 
Anterior GB wall thickness was measured, and $3 \mathrm{~mm}$ wall thickness was taken as upper limit of normal in adequately distended gall bladder. Thickening caused by intrinsic GB pathologies like cancer and acute cholecystitis were excluded. The relationship of the GB wall thickening with ascitic echotexture determined by ultrasound is presented in Table 5. The majority of patients (71\%) with transudate showed GB wall thickening, but only $10 \%$ in exudative ascites. Similarly, when compared with disease categories, GB wall thickening was observed in $15(93.8 \%)$ out of 16 patients with benign causes, whereas it was found in $1 / 20$ $(5 \%)$ of malignant causes $(\mathrm{P}$ value $=0.000)$.

In the majority of the cases, $52(85.2 \%)$, the distribution of ascites was in greater sac, and the remaining $9(14.8 \%)$ were in both compartments. Among 9 cases, 7 were cancer cases and 2 were caused by tuberculous peritonitis. Both tuberculous peritonitis cases showed multifocal loculation in the greater sac. Lymphadenopathy was seen in 12 cases, and it was detected in 10 cancer cases while 2 were associated with tuberculous peritonitis.

Table 5: Crosstabulation of sonographically determined ascites pattern with GB wall thickening in 61 patients at TASH, 2017.

\begin{tabular}{llllll}
\hline \multirow{2}{*}{$\begin{array}{l}\text { US determined ascites } \\
\text { pattern }\end{array}$} & \multicolumn{2}{c}{ GB wall thickening } & Total & P value \\
\cline { 2 - 5 } & & No & Yes & $31(100.0 \%)$ & \\
\cline { 2 - 5 } Total & Transudate & $9(29.0 \%)$ & $22(71.0 \%)$ & $30(100.0 \%)$ & 0.000 \\
\hline
\end{tabular}

\section{DISCUSSION}

This study was aimed to assess the role of transabdominal ultrasonography in characterizing and determining the etiology of ascites in comparison with laboratory ascitic fluid analysis and other methods used to establish the final diagnosis.

In our case series, cancer was the commonest cause, 20(32.8\%), of ascites and the second cause was chronic liver disease 9(14.8\%). This finding was contrary to causes other studies done in developed and developing countries. For instance, the Sudanese study by Alnumeri et al. revealed that the most common etiologies of ascites in Sudanese patients was liver cirrhosis followed by cancer (11). Similarly, Khan showed that cirrhosis was the commonest cause (about $60 \%$ ) of 104 Quatrain patients (9). The study on westerns is consistent with those studies, showing that cirrhosis was the cause of ascites in $75 \%$ of cases (13). The reason of the dominance of cancer patients in our case series can be attributed to the fact that TASH is the only hospital in the country that gives oncology service. Thus, all cancer cases all over the country are referred to this center.
Most of them are bound to be referred to Radiology Repartment for imaging study to further establish the diagnosis and determine the staging before initiating chemotherapy and radiotherapy.

Out of 59 cases in this case series, ultrasound suggested causes of ascites as benign or malignant in 54(91.5\%) cases. This was possible by combining multiple intra-abdominal sonographic findings. These findings include morphological changes in the abdominal organs and peritoneal layers. Moreover, assessing the presence or absence of lymphadenopathy, vascular patency and GB wall thickening helped in suggesting the likely causes. Topel et al. compared US and CT in detecting those radiological signs that help in suggesting the etiology of ascites as benign or malignant. He concluded that US was significantly superior to CT scan in evaluation of GB wall thickening, but no significant difference was identified between the two methods in the evaluation of the remaining parameters (2). This implies that ultrasound has comparative efficacy with $\mathrm{CT}$ in suggesting the etiology of ascites.

DOI: http://dx.doi.org/10.4314/ejhs.v29i3.11 
In this study, transabdominal Ultrasound was able to detect as little as about $14 \mathrm{ml}$ of ascites at a single location using high frequency linear transducers. In a study done in 1970 by Goldberg et al, after introducing a known amount of fluid, determined the amount detected by ultrasound. They showed that ultrasound was able to detect about $100 \mathrm{ml}$ instilled fluid (6). However, it should be noted that they did not mention the amount detected at a single spot by means of ultrasound.

As shown in Table 4, from grades 0 to 2 and 3 to 4 were lumped for the purpose of statistical comparison to see if there is any relationship with grouped ascitic etiologies. As shown on Table 4, there was no significant relationship between the ascitic volume grades and categorized etiology of ascites. ( $\mathrm{P}$ value $=0.573$ ). Similarly, no relation was observed between ascitic volume grades and ascitic pattern determined with US and SAAG (P value $=0.520 \&$ P. value $=0.973$, respectively $)$. In contrast to our findings, the study done by Alnumeiri et al. who used a similar grading system, showed that there was a significant correlation between ascitic volume and SAAG level. He concluded that when the SAAG value increases, the volume also correspondingly increases (11). The variation might be due to the delay of our cancer patients until they were referred to the only oncologic center in the country. In that situation, the cancer patients likely develop an increased amount of ascites.

GB wall thickening was seen in $15(93.8 \%)$ of 16 benign cases while only $1(5 \%)$ among 20 cases of malignant cause showed this finding $(\mathrm{P}$ value $=0.000)$. Of course, this excludes intrinsic causes of GB thickening.

This is in agreement with other studies which revealed a significant correlation between ascites caused by benign systemic causes and GB wall thickening. Tsujimoto et al., for example, in their study on 65 patients with benign and malignant causes of ascites, ultrasound showed normal GB wall thickness $(\leq 3 \mathrm{~mm})$ on 37 patients and increased wall thickening $(>3 \mathrm{~mm})$ with double wall appearance in 28 patients. Of the 37 patients with normal GB wall thickening, 35(95\%) had peritoneal carcinomatosis. Of the 28 patients with thickened double-walled GBs, 23(82\%) had benign disease. Benign causes listed in his study include cirrhosis, nephrotic syndrome and hypoalbuminemia (14). Topal et al. also concluded that GB thickening had sensitivity and specificity of 80 and 88 in distinguishing benign and malignant causes of ascites, respectively (2).
The US classification of ascites as exudate and transudate overall agreement to SAAG was $83.1 \%$ of the cases. However, if we see each disease category, US agrees with SAAG in $16(94.1 \%)$ out of 17 benign causes, $15(75 \%)$ out of 20 malignant causes, and $5(83.3 \%)$ out of 6 inflammatory cases. This finding is consistent with other studies. Alnumeri et al. showed that there was $100 \%$ agreement between US characterization and SAAG results in benign pathologies, $77.8 \%$ in cancer cases and $87.5 \%$ in inflammatory cases (11). Similarly, Edell et al studied the accuracy of ultrasound in distinguishing transudative and exudative ascites on 65 patients. Ultrasound was able to identify all 50 transudative patients and 11 of 15 exudative ascites giving accuracy of $100 \%$ and $73.3 \%$, respectively (15).

There were about 17 patients with mixed etiologies of ascites defined in our study. This group which was included in our classification of causes of ascites showed the least agreement with ultrasound characterization of ascites with disease categories. This is attributable to the combined nature of the causes of ascites expected to give either transudative or exudative ascites if they occur in isolation. That in turn, dictates the type of ascites observed on ultrasound and laboratory analysis. It can be either transudate or exudate depending on the dominant cause. For example, in this study, among 10 cases of $\mathrm{HCC}$ on the background of cirrhotic liver, $9(90 \%)$ of them had transudative ascites on SAAG and $7(70 \%)$ on US evaluation. It was only one case who had exudative ascites on both methods.

Similarly, we expect inflammatory pathologies to give exudative ascites. However, when they are combined with hypoproteinemia, the sonographic and mainly laboratory characteristics of ascites certainly will change. Therefore, these cases also are included under mixed causes. For instance, in our two tuberculosis patients, the ascites was understandably expected to be exudative type, but when associated with hypoalbuminemia or adult malnutrition the result became transudate.

As far as we know, the mixed group has never been recognized as such on imaging studies of ascites. However, literatures that deal with laboratory ascitic fluid analysis frequently mention that the mixed group more often show a transudative type of ascites $(7,10)$.

There were some limitations in our study. In some cases of our series, the final diagnosis of etiology of ascites was done on the basis of the 
combination of various imaging, clinical and laboratory data. It was impossible to get pathological diagnosis for all cases. Furthermore, no correlation of ultrasound findings with surgical findings were done in our series, as most of the cases were not candidates for surgery. The reason is that the majority of cases were advanced cancer cases.

In conclusion, ultrasound is a reliable, noninvasive and cheaper means of detecting, characterizing and quantifying ascites. It has a significant accuracy in distinguish transudate and exudate ascites and suggesting the underlying cause. It can be a valuable method of investigation of ascites in places where CT and MRI are not available. It is the best complement for laboratory investigation of ascites in suggesting the diagnosis. It can even serve as a substitute to characterize ascites with a significant degree of certainty where there is no adequately equipped laboratory unit.

\section{ACKNOWLEDGEMENTS}

We would like also to extend our special appreciation for Dr. Wondiwossen Amogne and Dr Amare Mengistu for their continuous guidance and unreserved support from the process of developing the proposal to analysis of the result. We are also indebted to radiology residents for their unceasing help during the process of data collection.

\section{REFERENCES}

1. Hanbidge A.E, Lynch D, Stephen RW. US of the peritoneum. Radiographics. 2003; 23(3): 663-84.

2. Naile B. Topal, Selim G, Ilker E, Gursel S. The role of ultrasonography and computed tomography in determining the etiology of ascites. Saudi Med J 2007; 28(12):1822-6.

3. Khaladkar SM, Aditi G, Dhaval T, et.al. Differentiation of malignant and benign ascites by Ultrasonography and/or CT. International $\mathrm{J}$. of Healthcare and Biomedical Research, 2015 Jan; 3 (2): 102-116.

4. Gupta R, Misra SP, Dwivedi M, Misra V, Kumar S, Gupta SC. Diagnosing ascites: value of ascitic fluid total protein, albumin, cholesterol, their ratios, serum-ascites albumin and cholesterol gradient. J Gastroenterol Hepatol. 1995; 10: 295-299.

5. Cattau E, Benjamin S, Knuff T, Castell D. The accuracy of the physical examination in the diagnosis of suspected ascites. JAMA. 1982; 247: 1164-1166.

6. Barry BG, Gerald AG, Harris RC. Evaluation of ascites by ultrasound. Radiology. 1970 July; 96(1): 15-22.

7. Bruce AR, Agnes AM, Evangelos AA, Mainor RA, Michelle AI, John GM. The serum-ascites albumin gradient is superior to the exudate-transudate concept in the differential diagnosis of ascites. Ann Intern Med. 1992; 117(3): 215-20.

8. Tarn AC, Lapworth R. Biochemical analysis of ascitic (peritoneal) fluid: what should we measure? Annals of Clinical Biochemistry. 2010; 47: 397-407.

9. A. Albillos, V.Cuervas-Mons, I. Millan, et al. Ascitic Fluid Polymorphonuclear Cell Count and Serum to Ascites Albumin Gradient in the Diagnosis of Bacterial Peritonitis. Gastroenterology. 1990; 98:134-140.

10. Khan, FY, Ascites in the state of Qatar: aetiology and diagnostic value of ascitic fluid analysis. Singapore Med J. 2007; 48(5): 434.

11. Alnumeiri MS, Ayad $\mathrm{CE}$, Ahmed $\mathrm{BH}$, Abdalla EA. Evaluation of Ascites and its Etiology Using Ultrasonography. J Res Development. 2015; 3(1): 119.

12. Harald Lutz EB. WHO manual of diagnostic ultrasound. 2nd ed. Vol. 1. Malta; Gutenberg Press Ltd.; 2011. Chap 2, Examination Technique: General Rules and Recommendations; page 29-30.

13. Hirra T, Huda S, Bushra AS. Causes, management and complications of ascites: a review. International Current Pharmaceutical Journal. 2015 February; 4(3):370-377.

14. Tsujimoto F, Miyamoto Y, Tada S. Differentiation of benign from malignant ascites by sonographic evaluation of gallbladder wall. Radiology. 1985; 157(2):503-4.

15. Edell SL, Warren BG. Ultrasonic differentiation of types of ascitic fluid. AJR Am J Roentgenol. 1979 July; 133:111-4. 